

\title{
Behavioral Simulation Model for SFRC and Application to Flexural Fatigue in Tension
}

\author{
Xuejuan Tang ${ }^{1}$, Xuehui $\mathrm{An}^{2 *}$ and Koichi Maekawa ${ }^{3}$
}

\begin{abstract}
A fatigue behavioral simulation for steel fiber reinforced concrete (SFRC) subjected to high cycle repetition of loads is presented. The fatigue constitutive models for normal strength concrete developed in the past decades are extended to those for SFRC with regard to tension, compression and shear transfer along dispersed cracking. The hysteretic path-dependency of SFRC is extensively focused on as well as post-cracking tension softening, because it greatly affects the stress-strain amplitudes of SFRC inside structures. The interaction of multi-directional cracks is taken into account based upon the fixed crack approach for enabling the damage evolution under the principal stress rotation. The rate effect on the stress and strain relation is also formulated to take into account the nonlinearity related to both loading rates and numbers of cycles. These proposed models for SFRC are experimentally verified in view of S-N diagrams of flexural tension for practical use.
\end{abstract}

\section{Introduction}

Steel fiber reinforced concrete (SFRC) is a cementbased material in which short steel fibers are dispersed and distributed randomly. It's generally agreed that the principal beneficial effects of fibers emerge after the crack initiation due to the bond or bridging effect of steel fibers across cracks in concrete matrix. This benefit makes steel fibers significantly improve mechanical behaviors of concrete matrix such as ductility, durability, energy absorption capacity, toughness and increase in resistance against cyclic and dynamic loads (ACI 544.1 R-96; Shah 1991; Naaman and Gopalaratnamt 1983; Sumitro and Tsubaki 1998; Subramaniam et al. 2002). Then, SFRC has been practically used in structural engineering, namely, pavements and overlays on bridge decks, tunnel linings (Nanakorn and Horii 1996), repairing and strengthening works.

The use of this concrete system such as pavements and overlays requires high performance to assure its fatigue safety during long-term service life. Consequently, there were plenty of experimental investigations on flexural fatigue of SFRC in the past decades, mainly focusing on the parameters of steel fibers volume contents, types of fiber and the aspect ratio (Chang and Chai 1995; Nanni 1992; Singh and Kaushik 2001; Zhang et al.

${ }^{1}$ Postdoc, Department of Hydraulic Engineering, Tsinghua University, Beijing, China and PowerChina Chengdu Engineering Corporation Limited, Chengdu, China.

${ }^{2}$ Profesor, Department of Hydraulic Engineering, Tsinghua University, Beijing, China.

*Corresponding author,

E-mail: anxue@tsinghua.edu.cn

${ }^{3}$ Department of Civil Engineering, The University of Tokyo, Tokyo, Japan.
2000; Zhang and Stang 1998). These experimental studies under certain stress amplitudes may have led to some empirical fatigue formulations for design in terms of S-N diagrams. However, these empirical formulas may have some difficulties in applying for nonlinear analyses to trace the full paths of stresses and strains at each location of structural members to failure, because stress and strain amplitude may vary irregularly in SFRC applications under real moving traffic loads.

Similar processes to fatigue failure have been reported between SFRC and normal strength concrete. That is to say, fatigue failure usually starts from the initially inherent flaws such as pores, air voids, lenses of bleed water under coarse aggregates and shrinkage cracks. During cyclic loading, repeated stress makes these initial flaws interconnected and micro-scaled crack may propagate. Cumulative damage occurs in bond between aggregates and mortar, and the stressed area gradually decreases eventually resulting in fatigue failure. It is empirically known that the shrinkage of concrete may accelerate the fatigue damage evolution (Fujiyama et al. 2013).

However, due to the bridging effect, steel fibers may arrest crack propagation and help concrete absorb more energy, which leads to better post-cracking softening (Wecharatana and Shah 1983). During the hysteretic path tests, the presence of fibers was found not only to retard the crack propagation but also crack closing. And more residual strain can be found (Gopalaratnam and Shah 1987) in cyclic loading experiments as shown in Fig. 1. This may cause different amplitude of deformation inside SFRC from that of normal structural concrete.

In the past decades, much progress can be seen on fatigue behavioral simulation of normal strength concrete as well. Nonlinear fatigue models before and after cracking (Maekawa et al. 2006) has been developed and experimentally verified by numerically tracing the mi- 
croscopic material states at each moment and location on the whole loading paths to failure. These models were formulated in terms of tension, compression and shear along crack planes. In this paper, the authors try to extend this framework of normal strength concrete to those for SFRC, and to aim at fatigue behavioral simulations for SFRC subjected to high cycle repetition of loads. The computed post-cracking response and fatigue life under constant amplitudes are expressed in the form of S-N diagrams of engineering use and verified experimentally.

\section{Methodology}

\subsection{Constitutive models for SFRC and} multi-directional cracking

In practice, the amount of steel fibers used is usually no more than $2 \%$ in concrete with coarse aggregates, because too much steel fibers may bring uneven mixing and placing problems (ACI 544.1R-96). Then, the scope of this study is so called SFRC having those properties and constraint stated above. Then, the modulus of elasticity and Poisson' ratio of SFRC is generally equal to those of a similar non-fibrous concrete (ACI 544. 1R-96).

Figure 2 shows a set of basic one-dimensional (1D) constitutive models of SFRC used in this paper. The two or three-dimensional extension $(2 \mathrm{D}, 3 \mathrm{D})$ of the modeling in Fig. 2 is performed by superimposing the 1D constitutive models on the basis of multi-directional cracking field as shown in Fig. 3 (Maekawa et al. 2003). Compared with normal concrete, the effect of added steel fibers on compressive strength is reported to be negligible, while considerable increase in ductility can be observed (ACI 544.1R-96; Nataraja et al. 1999). The authors direct their attention to the flexural tension where compressive nonlinearity is minor impact. Then in this study, the original normal concrete model (Maekawa et al. 2003) of elasticity, plasticity and damaging is directly adopted as shown in Eqs. (1)-(7) and Fig. 2-a, respectively.

1. Loading condition for $\varepsilon \leq \varepsilon_{c, \max }$

$$
\begin{aligned}
& \sigma_{c}=\omega K_{0} E_{c 0}\left(\varepsilon-\varepsilon_{p}\right) \\
& K_{0}=\exp \left\{-0.73-\frac{\varepsilon}{\varepsilon_{c}}\left[1-\exp \left(-1.25 \frac{\varepsilon}{\varepsilon_{c}}\right)\right]\right\} \\
& \varepsilon_{p}=\beta\left\{\frac{\varepsilon}{\varepsilon_{c}}-\frac{20}{7}\left[1-\exp \left(-0.35 \frac{\varepsilon}{\varepsilon_{c}}\right)\right]\right\} \varepsilon_{c} \\
& E_{c 0}=E_{0} \frac{f_{c}}{\varepsilon_{c}}
\end{aligned}
$$

2. Reloading condition for $\varepsilon_{c, \max }<\varepsilon \leq \varepsilon_{c, 0}$

$$
\sigma_{c}=\omega\left[\sigma_{c, \text { max }}-\left(\sigma_{c, \text { max }}-\sigma_{c, 0}\right) \frac{\varepsilon_{c, \text { max }}-\varepsilon_{p}}{\varepsilon_{c, \text { max }}-\varepsilon_{c, 0}}\right]
$$
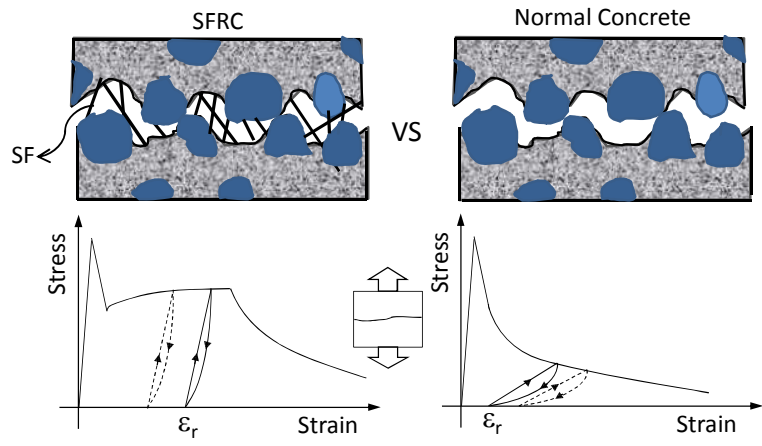

Fig. 1 Different mechanism of fibers on crack closure for SFRC and normal concrete.
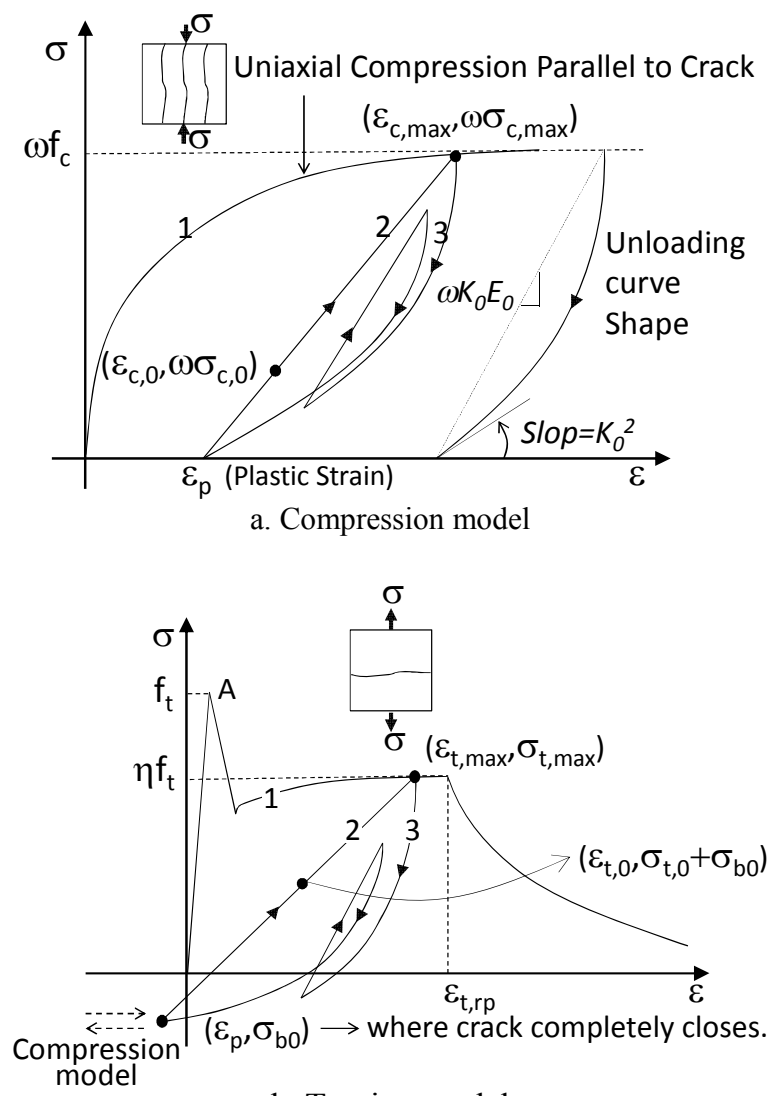

b. Tension model

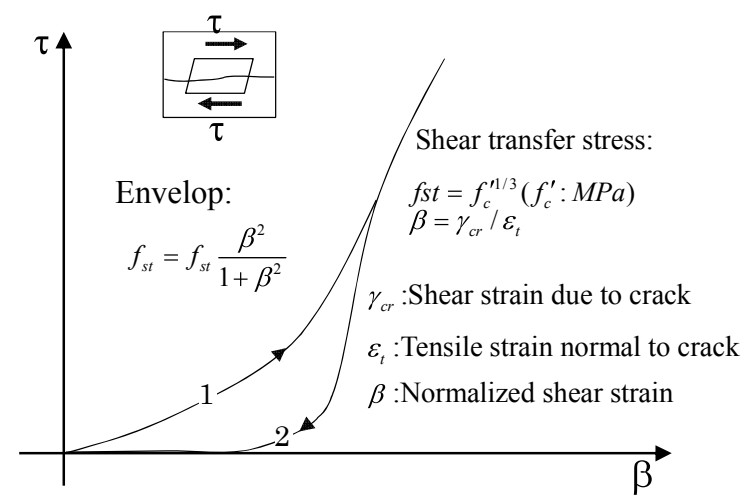

c. Shear transfer model

Fig. 2. Basic constitutive models for SFRC. 
3. Unloading condition for $\varepsilon_{c, 0}<\varepsilon, \varepsilon>\varepsilon_{c, \max }$

$$
\begin{aligned}
& \sigma_{c}=\omega K_{0} E_{c 0}\left(\varepsilon-\varepsilon_{p}\right) \alpha \\
& \alpha=\operatorname{slop}+\left[\frac{\sigma_{c, 0}}{K_{0} E_{c 0}\left(\varepsilon-\varepsilon_{p}\right)}-\operatorname{slop}\right]\left(\frac{\varepsilon-\varepsilon_{p}}{\varepsilon_{c, 0}-\varepsilon_{p}}\right)^{P N}
\end{aligned}
$$

where, $\sigma_{c}$ and $\varepsilon$ is the concrete compressive stress and strain, $\omega$ is compressive strength reduction factor due to transverse cracking, $K_{0}$ indicates the fracture parameter of concrete, $E_{c 0}$ and $E_{0}$ mean the initial stiffness and coefficient as 2.0, $\varepsilon_{c}$ and $\varepsilon_{p}$ mean the compressive peak strain and plastic strain, $\varepsilon_{c, \max }$ and $\sigma_{c \text {, denote the }}$ maximum compressive strain and the corresponding strain, $\sigma_{c, 0}$ and $\varepsilon_{c, 0}$ mean the current compressive stress and strain, slop and $P N$ are unloading parameters and determine the shape of the unloading curve as shown in Fig. 2-a, slop $=\mathrm{K}_{0}^{2}$ and $P N=2.0 . \beta$ is the strain-rate factor, $=1$ for dynamic and $=1.5 \sim 2$ for static analyses.

As stated previously, many researches efforts have been directed to nonlinear tensile behaviors of SFRC by considering steel fibers related parameters such as the volume content, geometry and its aspect ratio. According to composites material mechanics, Naaman (1995) reported that different volume contents may bring about different post-cracking behaviors of SFRC and about $2 \%-3 \%$ is the critical level via theoretical analysis. Below the critical level, SFRC exhibits non-multiple but localized single cracking and could be considered as quasi-brittle material like plain concrete. However, in contrast, multiple cracking and strain hardening performance was greatly innovated by $\mathrm{Li}$ (2003) with non-steel fibers. Among these challenging studies in the past, the authors intend to cover just quasi-brittle SFRC in this paper to clarify the scope of the modeling proposed.

Actually, related experimental studies (ACI 544.1R-96) in pure uniaxial tension indicate that SFRC in practice (usually with less than $2 \%$ volume content) exhibits non-multiple but single cracking. Generally as shown in Fig. 2-b, contribution of steel fibers under tension roughly performs following three stages:

(1) Stage 1: Steel fibers and concrete almost keep with the same deformation before cracking of concrete matrix. Point A means concrete cracks and simultaneously arrival at the peak load.

(2) Stage 2: After cracking, steel fibers begin to play a major role owing to bridging of steel fibers across cracks. With the crack opening, contribution of steel fibers increases till the end of the bridging by steel fibers.

(3) Stage 3: Bridge effect decreases resulting from pullout of steel fibers, and stress gradually decays.

Furthermore, SFRC exhibits the different unloading paths compared to normal strength concrete. Based on the discussion in reference to the normal concrete constitutive model (Maekawa et al. 2006), the authors in this paper propose the cyclic tension model of SFRC, which are simply expressed by Eqs. (8)-(15).

1. Loading condition for $\varepsilon \geq \varepsilon_{t, \max }$

$$
\begin{aligned}
& \sigma=E_{0} \varepsilon, \text { for } 0 \leq \varepsilon<\varepsilon_{t, c r} \\
& \sigma=\alpha_{1} f_{t} \varepsilon^{\beta_{1}}, \text { for } \varepsilon_{t, c r} \leq \varepsilon<\varepsilon_{t, r p} \\
& \sigma=\alpha_{2} f_{t} \varepsilon^{\beta_{2}}, \text { for } \varepsilon_{t, r p}<\varepsilon
\end{aligned}
$$

2. Reloading condition for $\varepsilon_{t, 0} \leq \varepsilon<\varepsilon_{t, \max }$

$$
\begin{gathered}
\sigma=\left\{\sigma_{t, \max }-\left[\left(\sigma_{t, \max }+\sigma_{b 0}\right)-\sigma_{t, 0}\right] \frac{\varepsilon_{t, \max }-\varepsilon_{p}}{\varepsilon_{t, \max }-\varepsilon_{0}}\right\}-\sigma_{b 0} \\
\sigma_{b 0}=\sigma_{t}\left(0.05+0.15 \frac{\Delta \varepsilon_{t, \max }}{5 \varepsilon_{t, c r}}\right)>0.2 \sigma_{t, u}
\end{gathered}
$$

3. Unloading condition for $\varepsilon<\varepsilon_{t, 0}, \varepsilon<\varepsilon_{t, \max }$

$$
\begin{aligned}
& \sigma=E_{b 0}\left(\varepsilon-\varepsilon_{p}\right) \alpha+\sigma_{b 0} \\
& \alpha=\operatorname{slop}+\left[\frac{\sigma_{t, 0}}{E_{b 0}\left(\varepsilon-\varepsilon_{p}\right)}-\operatorname{slop}\right]\left(\frac{\varepsilon-\varepsilon_{p}}{\varepsilon_{t, 0}-\varepsilon_{p}}\right)^{P N} \\
& E_{b 0}=\frac{\sigma_{t, \max }-\sigma_{b 0}}{\Delta \varepsilon_{t, \max }}
\end{aligned}
$$

where $\left(\alpha_{1}, \beta_{1}\right)$ and $\left(\alpha_{2}, \beta_{2}\right)$ are softening factors associated with the finite element size. Firstly, the relation of $\sigma$ (stress) and $\omega$ ( $\omega$ is a measured single crack width) can be obtained from the tension test. As single crack is observed in SFRC tension tests, which is similar to the case of normal strength concrete, the space-averaged strain in finite elements is calculated by $\varepsilon=\omega / L$ ( $L$ is the related finite element size) after cracking. Then Eqs. (9) and (10) can be settled. slop and $P N$ are unloading curve constants, slop $=0.0$ and $P N=6.0$ to describe the retarding of closing effect from flat crimped steel fibers in SFRC as stated in the previous section.

Here, two major parameters $\left(\eta, \varepsilon_{t, r p}\right)$ are employed to represent amount and length of steel fibers. For simplicity, the authors assume that: (1) the second peak strength coefficient $\eta$ is linear with the amount of steel fibers; (2) rupture strain $\varepsilon_{\mathrm{t}, \mathrm{rp}}$ is linear with the steel fiber length. For a certain type of steel fiber, these two parameters, which represent the stress-strain point starting the final softening as shown in Fig. 2-b, may be easily identified solely from the simple uniaxial tensile tests. In case of flexural tests, uniaxial tensile curve should be calculated from theoretical analysis. In future study, effect of different type of steel fiber would be taken into account by establishing the relationship of shape coefficient and fiber shape from sufficient experimental results.

For shear transfer along the crack orientation, two mechanisms are agreed in shear transfer of cracked concrete: fiber carrying shear across crack planes and 


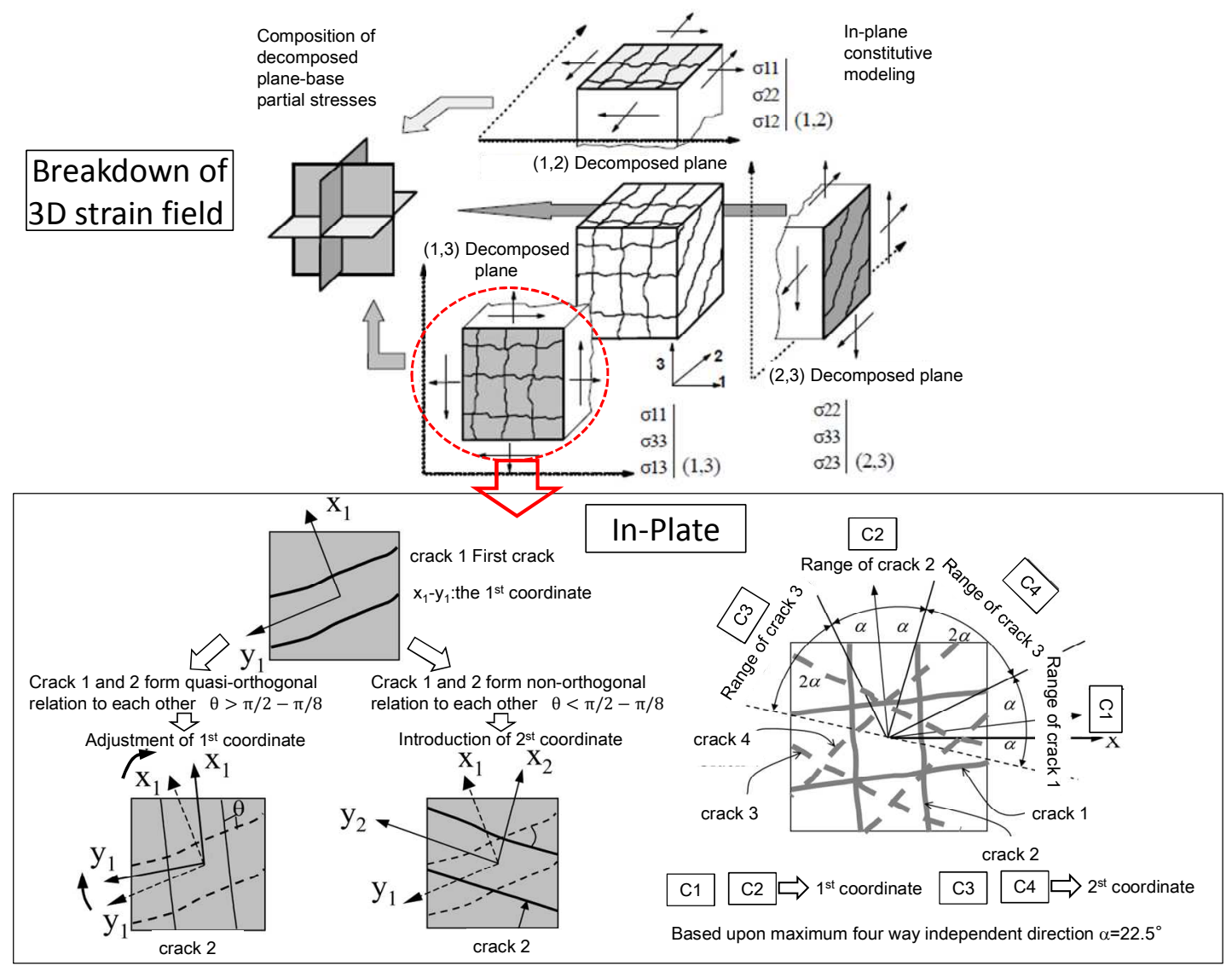

Fig. 3 3D crack element and Multi-directional fixed cracking method.

interlocking by rough crack surfaces' contact. Owing to the fiber inclusion, smaller individual crack width can be obtained under tension compared to normal strength concrete. Shear experiments of fiber reinforced concrete with and without coarse aggregates (Suryanto et al. 2010a, 2010b) indicates that coarse aggregates play a dominant role in crack shear transfer. Comparably, fibers' effect on the crack shear transfer is limited and negligibly small if coarse aggregates are included. Then, the contact density shear transfer model (Fig. 2-c), which was originally developed by $\mathrm{Li}$ and Maekawa (1987) for normal strength concrete, is applied to SFRC as well as,

$$
\begin{aligned}
& \tau=\int_{-\pi / 2}^{\pi / 2} \sigma_{c o n}^{\prime}(\theta) K(\omega) A_{t} \Omega(\theta) d \theta=\tau_{o r}(\delta, \omega) \\
& K(\omega)=1-e^{\left(1-\frac{0.5 G_{\max }}{\omega}\right)}
\end{aligned}
$$

where, $\tau$ means the transferred shear stress along the crack plane, $\delta$ means the shear slip along the cracks, and $\omega$ denotes crack width. $\sigma_{\text {con }}(\theta)$ is normal contact stress, $K(\omega)$ is the factor accounting for the maximum size of coarse aggregates denoted by $G_{\max } . A_{t}$ is the total area of crack surface per unit horizontal projection area. $\Omega(\theta)$ is the contact density function, $=0.5 \cos \theta$ for normal strength concrete. When $G_{\max }$ is larger than the maximum size of sand particle $(\approx 5 \mathrm{~mm})$ in normal strength concrete, $K(\omega)$ is expressed as Eq.(17). When we would tackle with fiber reinforced mortar without coarse aggregates, the shear transfer constitutive model has to be replaced with more appropriate one to consider the contribution of fibers in shear.

These 1D stress-strain relations of SFRC with cracks are applied to the stress carrying mechanism of three dimensions. For 3D finite element analysis, the general strain filed is decomposed into three in-plane sub-spaces (Maekawa et al. 2003) as shown in Fig. 3. For each sub-space, the multi-directional cracking method and constitutive laws of SFRC in two dimensions is employed.

\subsection{Extended fatigue models for SFRC}

For normal strength concrete, a cyclic fatigue structural analysis scheme of nonlinearity has been developed (Maekawa et al. 2006) and practically used for bridge maintenance (Fujiyama et al. 2013). In this high cycle fatigue analysis scheme, two major types of damages are described; time-dependent elasto-plastic and cyclic progressive damaging. Nonlinear fatigue constitutive models of concrete in tension, compression and shear were proposed as above. The microscopic material states at each moment and location on the loading path can be traced in the finite element analysis. On this framework, the mechanical damage and plasticity of concrete under high repetition of loads can be fully traced by logarith- 


\begin{tabular}{|c|c|c|}
\hline & $\begin{array}{l}\text { Constitutive models } \\
\text { of SFRC }\end{array}$ & $\begin{array}{l}\text { Enhanced models for high cycle } \\
\text { fatigue }\end{array}$ \\
\hline $\begin{array}{l}\text { Tension } \\
\text { model }\end{array}$ & 14 & $\begin{array}{l}K_{T} \text { is the tensile fracture parameter, which } \\
\text { stands for path-dependent instantaneous } \\
\text { fracturing, cumulative fatigue damage and } \\
\text { time-dependent tension creep. } \\
\mathrm{d} K_{T}=F d t+\mathrm{G} d \varepsilon+\mathrm{Hd} \varepsilon \\
\begin{array}{l}\text { Cyclic fatigue } \\
\text { damage }\end{array}\end{array}$ \\
\hline $\begin{array}{c}\text { Compression } \\
\text { model }\end{array}$ & $\underset{\substack{\varepsilon_{\mathrm{e}} \\
\text { (b) Compression model }}}{\sigma=\varepsilon_{e}+\varepsilon_{p}}$ & $\begin{array}{l}K_{C} \text { is the compression fracture parameter. } \\
\text { The nonlinearity of concrete is represented } \\
\text { by it. The time dependent plasticity, } \\
\text { fracturing and cyclic fatigue damage are } \\
\text { taken into account in parameter } K_{C} \text {. } \\
\mathrm{d} K_{\mathrm{c}}=\left(\frac{\partial K_{C}}{\partial t}\right) d t+\left(\frac{\partial K_{C}}{\partial \varepsilon_{e}}\right) \overrightarrow{d_{e}} \begin{array}{l}\text { Cyclic fatigue } \\
\text { damage }\end{array} \\
d \varepsilon_{p}=\left(\frac{\partial \varepsilon_{p}}{\partial t}\right) d t+\left(\frac{\partial \varepsilon_{p}}{\partial \varepsilon_{e}}\right) d \varepsilon_{e} \\
\text { (Mackawa and El- } \\
\text { Kachif } \text { et al. 2004) }\end{array}$ \\
\hline $\begin{array}{l}\text { Crack shear } \\
\text { model }\end{array}$ & (c) Shear model & $\begin{array}{l}\tau_{0} \text { is the original shear stress of concrete, } \\
\text { and represents the slip and width of crack, } \\
\text { and } X \text { is the accumulated path function, } \\
\text { which indicates the cyclic damage for shear } \\
\text { transfer. } \\
\tau=\mathrm{X} \tau_{0}(\delta, \omega) \quad \text { (Maekawa et al., 2006) } \\
\mathrm{X}=1-\frac{1}{10} \log _{10}\left\{\left(1+\int \mid d(\delta / \omega)\right\} \geq 0.1\right.\end{array}$ \\
\hline
\end{tabular}

Fig. 4 Constitutive fatigue models of concrete (Maekawa et al. 2006).

mic direct-path integration (Maekawa et al. 2008).

Hence, the cyclic fatigue analysis scheme of concrete proposed and verified by Maekawa and E1-Kashif (2004) are adopted as well (see Fig. 4). This section is to simply summarize this scheme as follows. The constitutive laws take into account the stress amplitude as a function of elastic strain to represent the substantial intensity of stress applied to "active" volume of materials. It is formulated in terms of uniaxial compression, tension, and shear transfer across crack, respectively as shown in Fig. 4.

The stress-strain relation of compressive concrete is modeled by elasto-plastic and fracture concept (Maekawa and E1-Kashif 2004) as:

$$
\begin{aligned}
& \varepsilon=\varepsilon_{e}+\varepsilon_{p} \\
& \sigma=E_{0} \varepsilon_{e} K_{c}
\end{aligned}
$$

The damage evolution due to repetition of load is implemented in fracture parameter $K_{c}$ as follows:

$$
d K_{c}=\left(\frac{\partial K_{c}}{\partial t}\right) d t+\left(\frac{\partial K_{c}}{\partial \varepsilon_{e}}\right) d \varepsilon_{e}
$$

$$
d \varepsilon_{p}=\left(\frac{\partial \varepsilon_{p}}{\partial t}\right) d t+\left(\frac{\partial \varepsilon_{p}}{\partial \varepsilon_{e}}\right) d \varepsilon_{e}
$$

where, $\varepsilon$ denotes total strain as a summation of elastic strain $\varepsilon_{e}$ and plastic strain $\varepsilon_{p}$. Total stress denoted by $\sigma$ is a product of initial stiffness $E_{0}$, elastic strain $\varepsilon_{e}$, and fracture parameter indicated by $K_{c}$. The nonlinearity of concrete is respected by $\varepsilon_{p}$ and $K_{c}$.

For tension nonlinearity, the stress-strain relation of tensile concrete is simply assumed by the elasto-plastic and damaging model as,

$$
\sigma=E_{0} \varepsilon K_{T}
$$

where, $\varepsilon$ is total strain and $K_{T}$ is a tensile fracture parameter for concrete. The evolution of fracture parameter $K_{T}$ is a scalar to represent path-dependent instantaneous fracturing, time-dependent tension creep and fatigue accumulative damage, formulated as:

$$
d K_{T}=H d \varepsilon+F d t+G d \varepsilon
$$

where, $H$ is the SFRC instantaneous fracture related to tension softening, as shown in Eqs. (8)-(10). The derivative of $F$ indicates time dependent fracture rate due to tension creep effect. The effect of addition of steel 


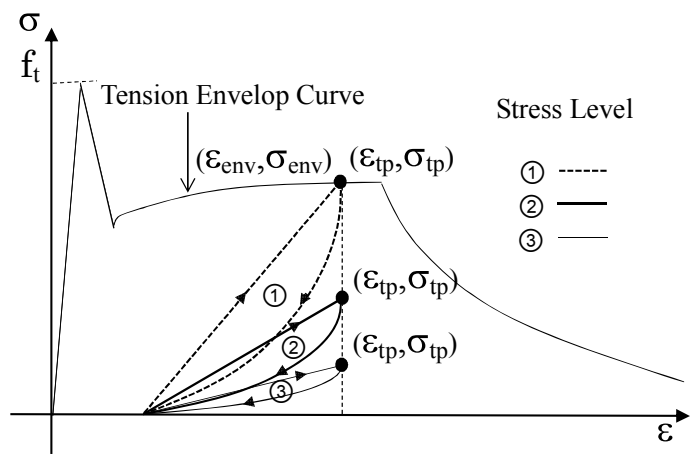

a. Fatigue cycle of different stress levels

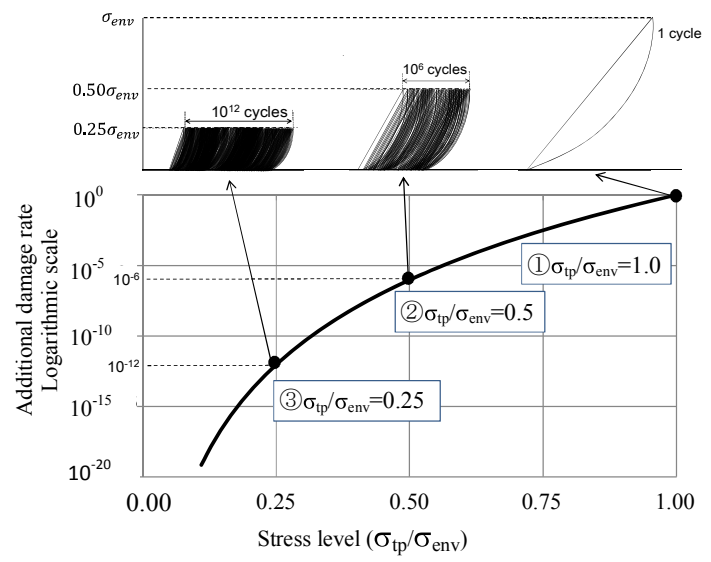

c. Additional damage rate under different stress levels

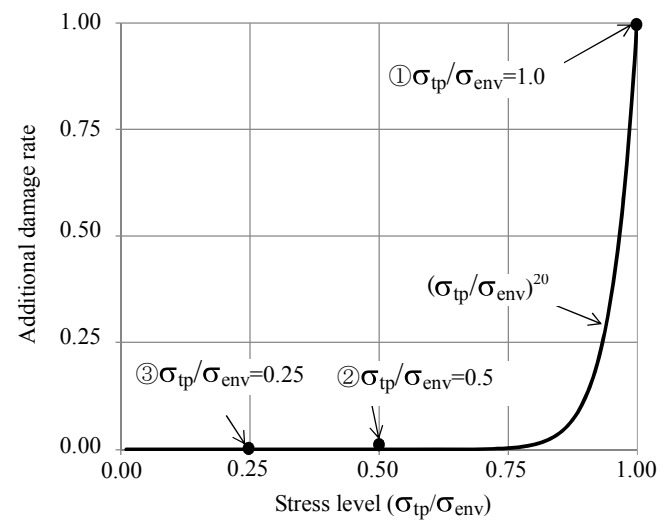

b. Damage rate in progress under different stress levels

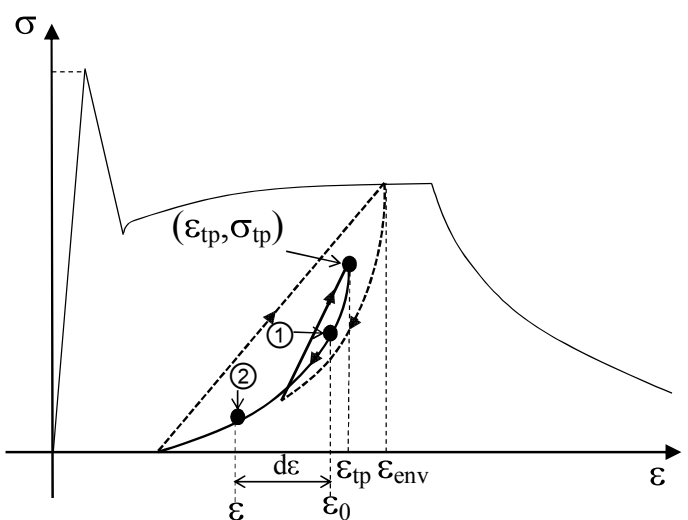

d. Additional damage and different strain amplitude

Fig.5 Cyclic fatigue damage due to repetition of load after cracking.

fibers on creep would be small or slightly increase from experimental results (Bentur and Mindess 1990; Balaguru and Ramakrishinan 1988), due to the fact that creep does not generally involve any macro cracking. Furthermore, $F$ is not a governing factor when permanent stress level is rather low like concrete bridge decks under the short-impulsive traffic loads to which this paper is directed. Then the formulas for normal concrete as Eqs. (24)-(26) are applied to SFRC simulation without modification in this study.

$$
\begin{aligned}
& F=-10^{-5} \cdot S^{3} \cdot\left(K_{T}-0.5\right)^{2}, \text { where } \varepsilon_{\max }<\varepsilon_{c r} \\
& F=-10^{-6} \cdot S^{6}, \text { where } \varepsilon_{\max } \geq \varepsilon_{c r} \\
& S \equiv \frac{E_{0} K_{T} \varepsilon}{f_{t}}
\end{aligned}
$$

where, $\mathrm{S}$ denotes the stress magnitude.

The derivative $G$ represents the cyclic fatigue cumulative damage due to repetition of loads after cracking, as shown in Eqs. (27)-(30).

$$
G d \varepsilon=K_{T}\left(\frac{\sigma_{t p}}{\sigma_{e n v}}\right)^{20} \cdot d \tilde{\varepsilon}
$$

$$
\begin{aligned}
& \sigma_{e n v}=\alpha f_{t} \varepsilon^{\beta} \\
& d \tilde{\varepsilon}=0, \text { when } d \varepsilon \geq 0 \\
& d \tilde{\varepsilon}=9 \cdot \gamma^{m} \cdot \frac{d \varepsilon}{\varepsilon_{0}}, m=8, \gamma \equiv-\frac{\varepsilon-\varepsilon_{t p}}{\varepsilon_{\max }}, \text { when } d \varepsilon<0
\end{aligned}
$$

where, $\sigma_{\mathrm{tp}}$ is the turning point stress indicating the fatigue stress level, $\varepsilon_{t p}$ is corresponding turning point strain of $\sigma_{\text {tp }}$ in one cycle, $\sigma_{\text {env }}$ means the stress corresponded to $\varepsilon_{\mathrm{tp}}$ on the envelope curve. Here, for non-cracked state, $\mathrm{m}=20$ and $\sigma_{\mathrm{tp}} / \sigma_{\mathrm{env}}=1.0$ is assumed.

In this study, the authors use the envelope and internal stress-strain curves for SFRC which is much different from those of normal strength concrete, but apply for the same fatigue damage accumulation law as that of normal concrete. This is the most highlight and original point of this study. Then, further explanation for fatigue damage accumulation law is added as follows.

The additional cyclic damage is related to both the stress level and the strain paths as denoted by Eq. (27). Here, let us focus on three complete cycles of different stress levels are shown in Fig. 5-a, where one-cycle damage evolution made by the stress level $[1]\left(\sigma_{\mathrm{tp}} / \sigma_{\text {env }}\right.$ $=1.0$ ) is expressed as unity. Equation 27 mathematically gives the damages under the different stress levels as 


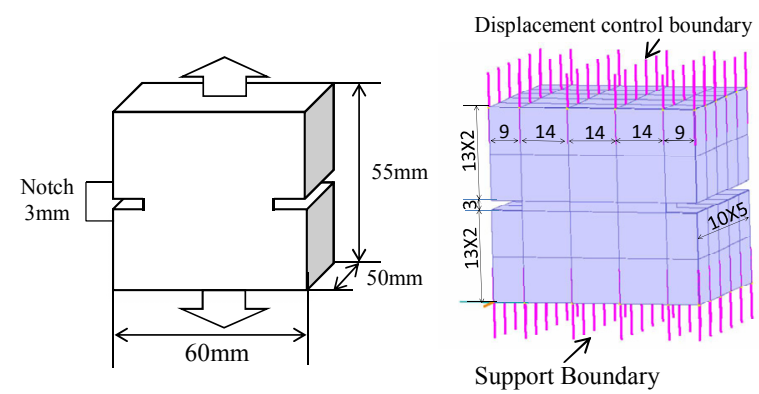

a. Geometry of specimen and finite element mesh

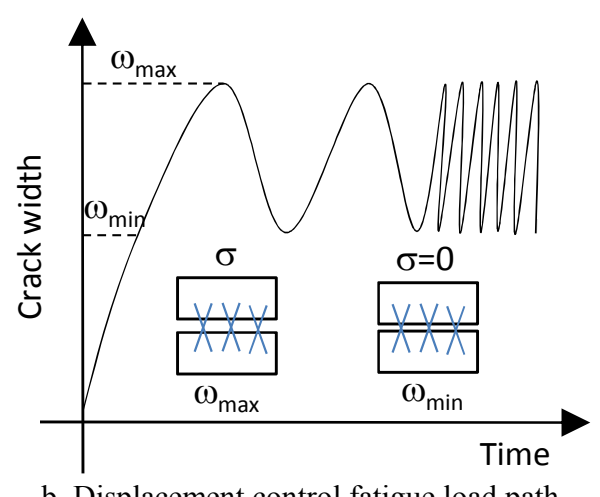

Fig. 6. Geometry and Load path.

shown in Figs. 5-b and 5-c. For stress level [2] $\left(\sigma_{\mathrm{tp}} / \sigma_{\mathrm{env}}=0.5\right)$, the additional damage rate of one cycle is about $10^{-6}$. That is to say, $10^{6}$ cycles of the stress level [2] $\left(\sigma_{\mathrm{tp}} / \sigma_{\mathrm{env}}=0.5\right)$ is equivalent to one cycle of $\left(\sigma_{\mathrm{tp}} / \sigma_{\mathrm{env}}\right.$ $=1.0$ ) in terms of damage accumulation. For the stress level $[3]\left(\sigma_{\mathrm{tp}} / \sigma_{\mathrm{env}}=0.25\right)$, it would be about $10^{12}$ cycles. The value of $d \tilde{\varepsilon}$ indicates the additional cyclic damage from different strain amplitude during unloading path. In this formulation, the cyclic damage is numerically integrated during the unloading path. The notation $\varepsilon_{0}$ and $\varepsilon_{\max }$ mean the current strain and historical maximum tension strains. As shown in Fig. 5-d, for strain path from [1] to [2], Eq. (30) gives assessment of the additional fatigue damage on this path. Then, different additional fatigue damage can be traced according to different stress level and strain amplitude.

The accumulated fatigue damaging law (Maekawa et al. 2006) as stated above may lead to the linearity on S-N diagram (Stress amplitude versus Number of cycles), which matches the experimental facts of SFRC (Johnston and Zemp 1991). However, the sensitivity of the stress amplitude on the fatigue life depends on the envelope stress represented by Equation (28) in the scheme of constitutive modeling for normal concrete as well as SFRC by Eq. (23). Then, the authors consider that the systematic verification in terms of derived S-N diagram is indispensable for verification of Eqs. (23) and (28), which controls the gradient of S-N diagram of SFRC.

Shear transferred across crack has two key mechanisms: crack surface and crack width as described by Eq. (16). The crack roughness and the contact friction are thought to be degraded after cyclic loads. Therefore, stiffness reduction factor is proposed to represent the fatigue damage on shear transfer. Based on basic contact density modeling, it is formulated (Maekawa et al. 2006) as,

$$
\begin{aligned}
& \tau=X \cdot \tau_{o r}(\delta, \omega) \\
& X=1-\frac{1}{10} \log _{10}\left[1+\int|d(\delta / \omega)|\right]
\end{aligned}
$$

Where $\tau$ is transferred shear stress under the high cycle load, and $X$ is the fatigue modification factor related with accumulation of shear deformation during the loading.

The factor $X$ changes in regard to common logarithmic scale. For example, when the true value of a common logarithm is null, factor $X$ is 1.0 and when the true value is $10^{9}$, factor $X$ is 0.1 . This indicates that reduction of shear stiffness expressed by factor $X$ is highly dependent on the number of load repetitions because the slip normalized width cannot increase in logarithmic for each cycle.

\section{Verification}

In order to verify the proposed models, two kinds of SFRC fatigue experiments without reinforced bars are selected. One is to trace the bridging stress degradation under constant amplitude of strain. The other is to simulate the S-N relationships of SFRC in flexure.

\subsection{Bridging stress degradation under fatigue loads}

Experimental results (Zhang et al. 2000) for determination of bridging stress degradation under uniaxial tension are adopted for verification. Geometry and finite element mesh are shown in Fig. 6-a. Hooked end steel fibers with $0.5 \mathrm{~mm}$ in diameter and $30 \mathrm{~mm}$ in length are used in concrete matrix with coarse aggregates. The volume content of steel fibers is $1 \%$. The specimens were subjected to uniaxial hysteretic-path tension with SFRC tension strength of 4.4MPa. In the fatigue test, firstly specimens were loaded to a certain maximum crack width, and then unloaded to zero load to keep a constant displacement control amplitude as shown in Fig. 6-b. According to the direct tension test and the finite mesh size, basic softening parameters can be identified for the softening stage (2) and (3). The values of $\alpha_{1}=0.63, \beta_{1}=0.078$ and $\alpha_{2}=0.032, \beta_{2}=-1.2$ correspond to Eqs. (9) and (10).

Figure 7 shows comparison of the experimental and analytical results of hysteretic-path test. As stated in the introductory section, practical application demand was taken into account. Therefore, in numerical analyses, the authors mainly focus on the crack width, which may experience for practical use of structures under fatigue service loads, and is less than $0.3 \mathrm{~mm}$ herein. Beyond this level of deformation, low cycle fatigue, which is out of scope of this paper, will be problematic and further 


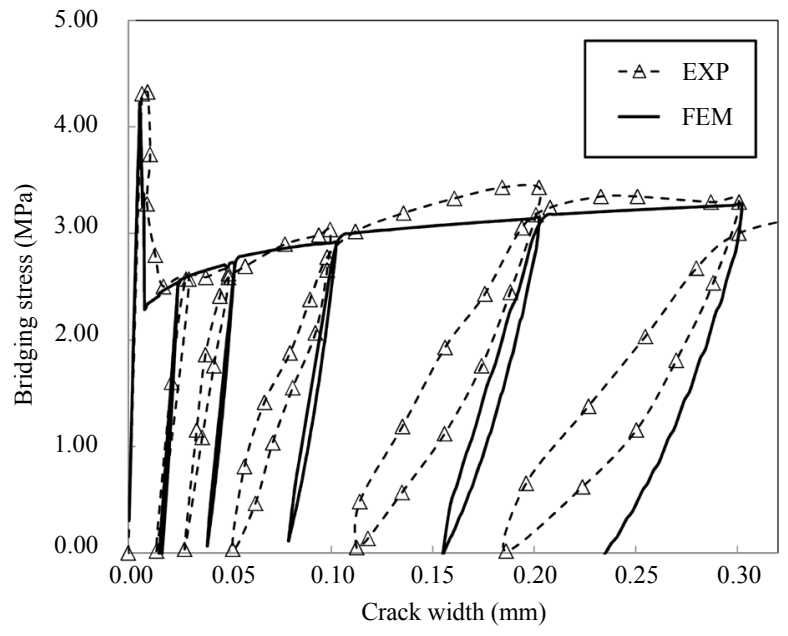

Fig. 7. Comparison of the experimental and analytical results of hysteretic-path test.

investigation will be challenged. A fine agreement could be found on envelop of stress-crack width curve. For unloading and reloading paths, when crack width is less than $0.1 \mathrm{~mm}$, simulation results can roughly catch the hysteretic paths. This result indicates that the proposed constitutive models can be applied to structural SFRC without reinforcing bars.

Figure 8 shows analytical bridging stress versus crack width relations of SFRC under fatigue loads of maximum crack width $\omega_{\max }=0.1 \mathrm{~mm}$ and $\omega_{\max }=0.05 \mathrm{~mm}$. As the magnified direct time-path integral (Maekawa et al. 2006) was called to accelerate computation, each loop of the bridging stress versus crack width represents the transient states of log-scale time, and the exact comparison of individual cyclic path is hardly conducted. Then, let us focus on an overall trend of the bridging stress decay for comparison of experiment and analysis. In fact, as cyclic fatigue loads were applied, the stiffness and bridging stress gradually degraded. For more detailed comparative analysis, the normalized bridging stress under specified fatigue cycles was calculated from analytical results as shown in Fig. 9. This figure
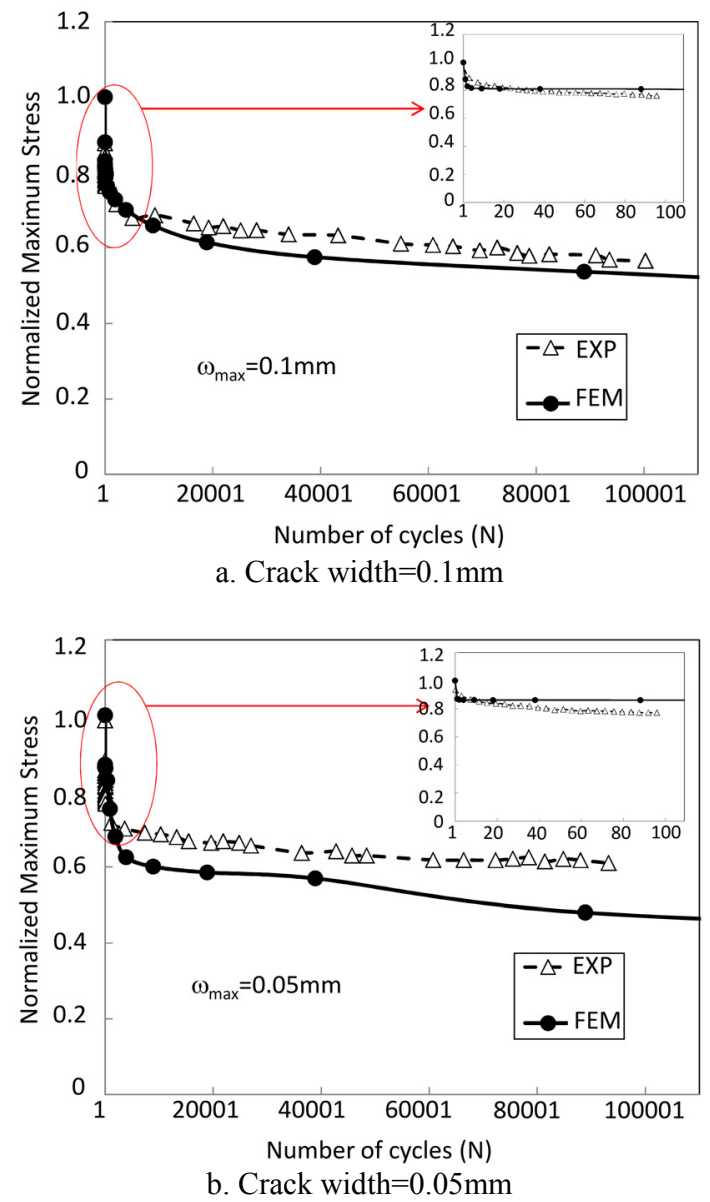

Fig. 9. Numerical and experimental results of Bridging stress and Number of Cycles.

shows the comparison of the analytical and experimental results of the SFRC specimens as well. A fair agreement can be found in the diagrams of bridging stress at different maximum crack width versus numbers of load cycles. This indicates the proposed fatigue models for SFRC based upon those for normal strength concrete can roughly trace the strength degradation caused by cumulative fatigue damage loss.

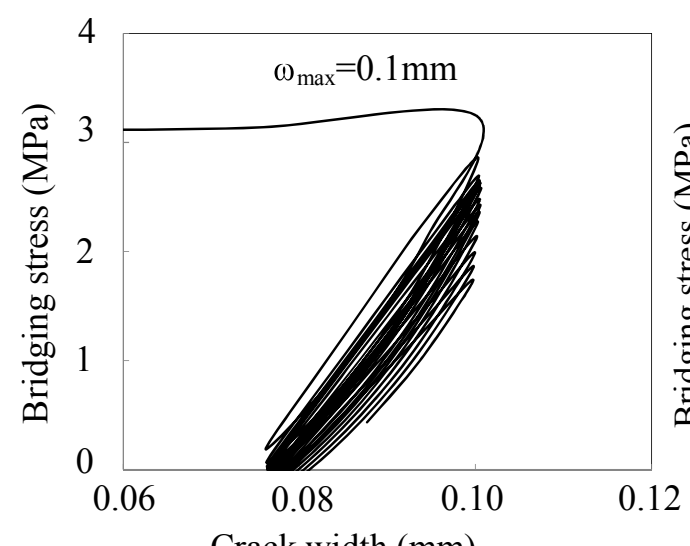

Crack width (mm)

a. Crack width $=0.1 \mathrm{~mm}$

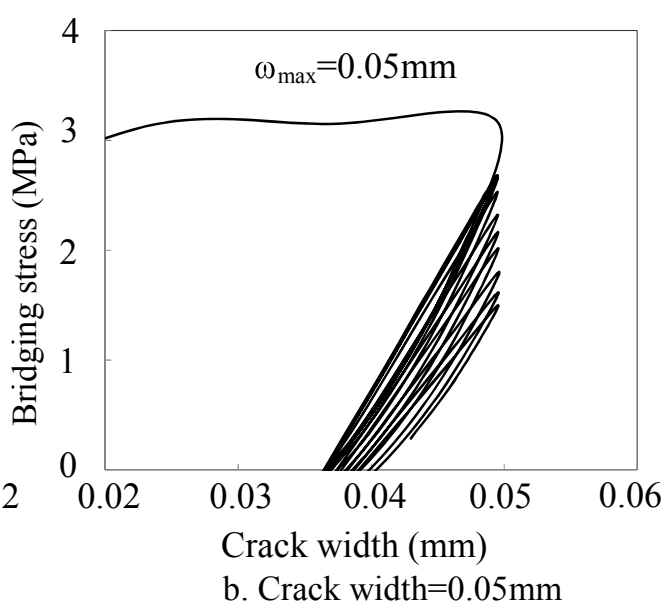

b. Crack width $=0.05 \mathrm{~mm}$

Fig. 8. Bridging stress and crack width relationship. 


\subsection{S-N diagram}

A number of investigations were carried out to study flexural fatigue performance of SFRC. S (fatigue stress level) and $N$ (number of cycles at failure) relation, which is known as Wohler equation, is widely adopted as one of methods to practically express the fatigue life of materials and structures. Here, a series of flexural fatigue experiments of SFRC conducted by Singh and Kaushik (2001) and Mohammadi and Kaushik (2005) are simulated by employing the proposed models. Firstly, SFRC specimens with 1\% volume content were numerically studied, and SFRC with different fiber lengths under the specified fiber content (1\%) were further discussed to verify the assumption that the rupture strain $\varepsilon_{t, r p}$ is linear with the fiber length.

Three cases marked as $\mathrm{A}, \mathrm{B}$ and $\mathrm{C}$ here, are with $1 \%$ volume content and with different fiber lengths. For case $\mathrm{A}$ and case $\mathrm{B}$, the softening relations were computed for the softening stage 2 and stage 3 as shown in Eqs. (33)- (36).

For case A (steel fiber with 50mm length):

$$
\begin{aligned}
& \sigma=1.5 f_{t} \varepsilon^{0.18} \\
& \sigma=0.02 f_{t} \varepsilon^{-0.7}
\end{aligned}
$$

For case B (steel fiber with $25 \mathrm{~mm}$ length):

$$
\begin{aligned}
& \sigma=1.7 f_{t} \varepsilon^{0.18} \\
& \sigma=0.0035 f_{t} \varepsilon^{-1}
\end{aligned}
$$

Fatigue flexural loads were applied on simply supported SFRC beams without notch. All beams are with size of $500 \mathrm{~mm} * 100 \mathrm{~mm} * 100 \mathrm{~mm}$. Flexural experiments were conducted at different stress ratio $\mathrm{S}\left(\mathrm{S}=f_{\max } / f_{r}\right.$, $f_{\min } / f_{\max }=0.1$, and $f_{r}$ is static flexural strength). Material properties of SFRC are summarized in Table 1.

Figure 10 shows the simulation of case A and B under static flexural loads. Before the first cracking, the higher stiffness can be clearly seen, but in experiment, the first crack strength of SFRC flexural test may not clearly appear in the load-displacement curve (ACI committee 544.2R-89 1999). The authors suppose that drying shrinkage induced micro-cracking close to the specimen surfaces may cause the lower stiffness and unclear cracking stress. Then, the authors mainly pay attention to the post-peak region which may have less to
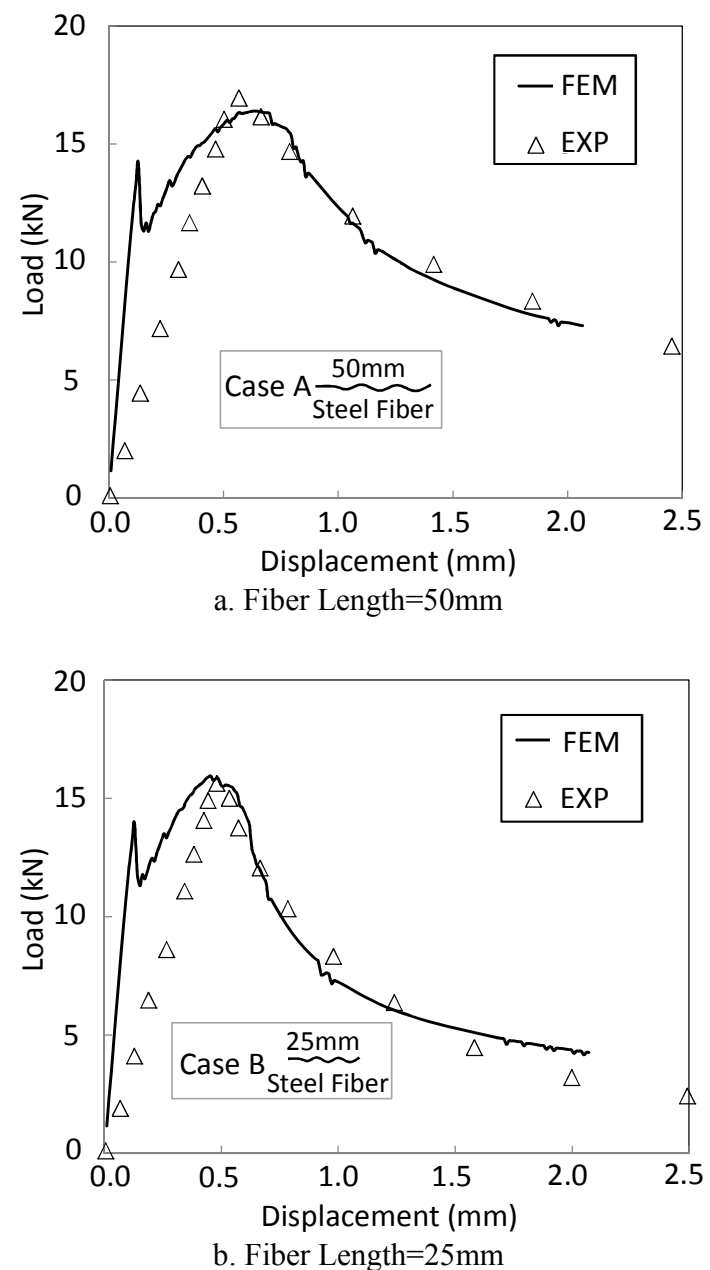

Fig. 10. Static flexural test simulation of Case A and B.

do with the drying shrinkage. The experimental and analytical comparison results indicate a fair consistency after the peak. By employing the proposed models, S-N diagrams from analysis are shown in Fig. 11. The experimental results after Weibull distribution statistical analysis are compared with FE analysis results. For both cases, experimental and numerical simulation results are not far from each other.

For case C, due to lack of the simple flexural curve, the authors cannot identify the monotonic tension envelope directly from related experiments. But, as stated previously in section 2.1, two key parameters are defined to describe SFRC tensile profile. Taking crimped

Table 1 Material property details.

\begin{tabular}{cccccc}
\hline Cases & Steel fiber Type & $V_{s f}$ & $\begin{array}{c}\text { Steel fiber } \\
\text { Length }(\mathrm{mm})\end{array}$ & $\begin{array}{c}f_{c}^{\prime} \\
(\mathrm{MPa})\end{array}$ & $\begin{array}{c}f_{t r}^{\prime} \\
(\mathrm{MPa})\end{array}$ \\
\hline $\mathrm{A}$ & $\begin{array}{c}\text { Flat } \\
\text { crimped }\end{array}$ & $1 \%$ & 50 & - & 7.5 \\
$\mathrm{~B}$ & & 25 & -16 & 7.16 \\
$\mathrm{C}$ & & 30 & 43.67 & 6.7 \\
\hline
\end{tabular}

$V_{s f}:$ is the volume content of steel fiber

$f_{c}^{\prime}$ and $f_{t r}^{\prime}$ : compression strength and flexural strength of SFRC

- : not recorded in literature 
steel fiber of this experiment for example, when volume content is $1 \%$ and $\mathrm{C} 40$ concrete, the second peak strength coefficient $\eta$ is about 0.62 . Then, rupture average strain $\varepsilon_{\mathrm{rp}}$ in mesh is about $5000 \mu$ for $25 \mathrm{~mm}$ length and about $7500 \mu$ for $50 \mathrm{~mm}$ length in mesh size of $25 \mathrm{~mm}$. According the assumption, relation of $\varepsilon_{\mathrm{rp}}$ and length of the fiber (L) can be obtained from a linear regression as shown in Eq. (37).

$$
\varepsilon_{t, r p}=a L+b
$$

where, $L$ is the length of fiber and notations ( $\mathrm{a}$ and $\mathrm{b}$ ) are coefficients from linear regression.

The fiber length of case $\mathrm{C}$ is $30 \mathrm{~mm}$, and its fiber content is $1 \%$. Therefore, it can approximately determine the second peak strength coefficient $\eta$ and rupture average strain $\varepsilon_{t, r p}$. The softening factors can also be computed: stage 2 as $\sigma=1.6 f_{t} \varepsilon^{0.18}$ and stage 3 as $\sigma=0.0035 f_{t} \varepsilon^{-1}$. By implementing the proposed fatigue analysis scheme, the $\mathrm{S}-\mathrm{N}$ diagram of case $\mathrm{C}$ is plotted as shown in Fig. 12. A reasonable consistence can be found.

The authors reported $1 \%$ volume content of fibers as a typical case where the mode of failure does not change regardless of stress amplitude. The larger or smaller quantities of fiber volume can be conducted similarly as well and apparent fair S-N diagram is possibly obtained as a macroscopic event. But in these cases, the mode of fatigue failure may vary, i.e., pullout of fibers from cement concrete matrix or fatigue rupture of fibers themselves. In these cases, the linearity of S-N curve might be lost. Then, for these cases, the model simulation shall be examined in terms of S-N diagram as well as microscopic mode of fatigue failure. This investigation will be reported in future.

\section{Conclusions}

Steel fiber reinforced concrete model applicable to high cycle fatigue is presented and the following conclusions are obtained.

(1) The envelope curve for the uniaxial tensile stress strain relation of SFRC, which greatly differs from that of normal strength concrete, is formulated according to the volume and shapes of fibers used.

(2) The internal curve for unloading and reloading cycles is found to be expressed with the same formulae as that for normal strength concrete by solely changing the parameters.

(3) Through the experimental verification, it is found that the formulae for high cyclic fatigue damage accumulation law of normal strength concrete is applicable to that of SFRC without any modification no matter how greatly the envelope curves differ.

\section{Acknowledgments}

The authors would like to express their gratitude to National High-tech R\&D Program of P. R. China (863 Program: No.2012AA06A112) and Open Research Fund

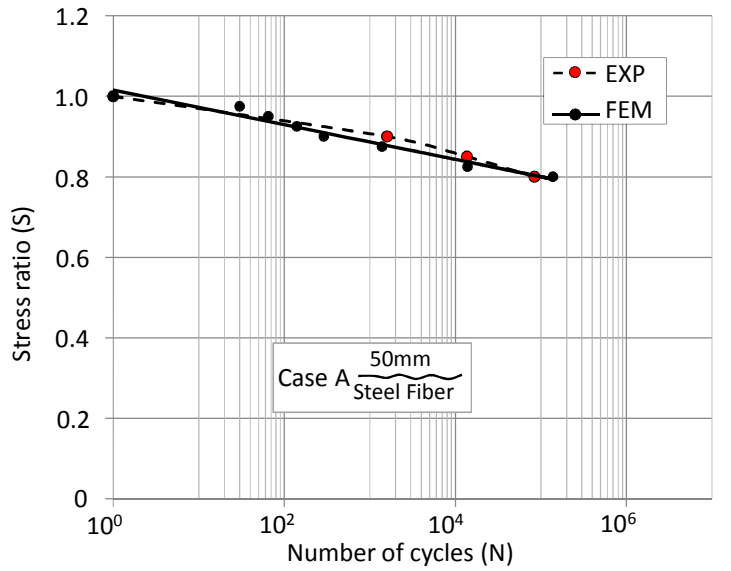

a. Fiber Length $=50 \mathrm{~mm}$

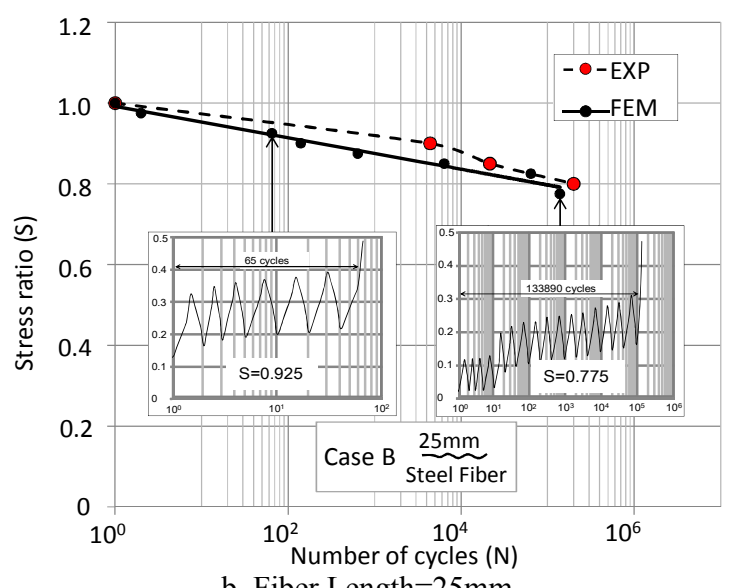

b. Fiber Length $=25 \mathrm{~mm}$

Fig. 11. Comparison of numerical and experimental results: Case A and B.

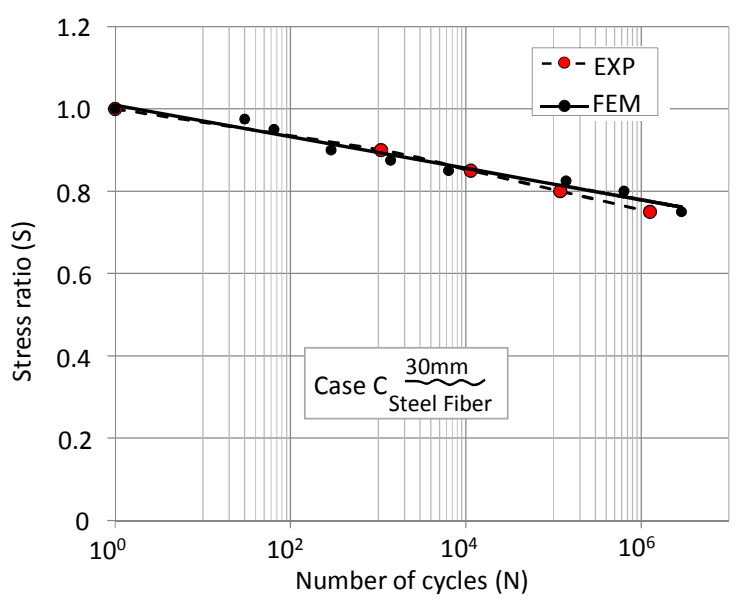

Fig. 12. Comparison result of Case $\mathrm{C}$ :Fiber Length $=$ $30 \mathrm{~mm}$

Program of State Key Laboratory of Hydro-science and Engineering under grant No.2012-Ky-02. The authors also appreciate the continuous discussion with Dr. C. Fujiyama of Hosei University.

\section{References}

ACI Committee 544.1R, (1996). "State-of-the-art report 
on fiber reinforced concrete." American Concrete Institute.

ACI Committee 544.2R-89, (1999). "Measurement of properties of fiber reinforced concrete." American Concrete Institute.

Balaguru, P. and Ramakrishnan, V., (1988). "Properties of fiber reinforced concrete: workability, behavior under long-term loading, and air-void characteristics." ACI material Journal, 85(3), 189-196.

Bentur, A. and Mindess, S., (1990). "Fiber Reinforced Cementitious Composites." Elsevier Appl. Sci., London.

Chang, D. and Chai, W., (1995). "Flexural fracture and fatigue behavior of steel fiber reinforced concrete structures." Nuclear Engineering and Design, 156(1-2), 201-207.

Fujiyama, C., Tang, X. J., Maekawa, K. and An, X. H., (2013). "Pseudo-cracking approach to fatigue life assessment of RC bridge decks in service." Journal of Advanced Concrete Technology, 11, 7-21.

Gopalaratnam, V. and Shah, S. P., (1987). "Tensile failure of steel fiber-reinforced mortar." Journal of Engineering Mechanics, 113(5), 635-652.

Johnston, C. D. and Zemp, R. W., (1991). "Flexural fatigue performance of steel fiber reinforced concrete-influence of fiber content, aspect ratio, and type." ACI Materials Journal, 88, July-August, 374-383.

Li, B. and Maekawa, K., (1987). "Contact density model for cracks in concrete." In: IABSE Colloquium Deft 87 on Computational mechanics of Concrete Structures, 51-62.

Li, V. C., (2003). "On engineered cementitious composites (ECC), A review of the material and its applications." Journal of Advanced Concrete Technology, 1(3), 215-230.

Maekawa, K. and E1-Kashif, K., (2004). "Cyclic cumulative damaging of reinforced concrete in post-peak regions." Journal of Advanced Concrete Technology, 2(2), 257-271.

Maekawa, K., Pimanmas, A., and Okamura, H., (2003). "Nonlinear Mechanics of Reinforced Concrete." Spon Press, London.

Maekawa, K., Toongoenthong, K., Gebreyouhannes, E. and Kishi, T., (2006). "Direct path-integral scheme for fatigue simulation of reinforced concrete in shear." Journal of Advanced Concrete Technology, 4(1), 159-177.

Maekawa, K., Fukuura, N. and Soltani, M., (2008). "Path-dependent high cycle fatigue modeling of joint interfaces in structural concrete." Journal of Advanced Concrete Technology, 6(1), 227-242.

Mohammadi, Y. and Kaushik, S. K., (2005). "Flexural fatigue-life distributions of plain and fibrous concrete at various stress levels." Journal of Materials in Civil
Engineering, 17(6), 650-658.

Naaman, A. E. and Gopalaratnamt, V. S., (1983). "Impact properties of steel fibre reinforced concrete in bending." Cement Composites and lightweight Concrete, 5(4), 225-233.

Naaman, A. E. and Reinhardt, H. W., (1995), "Characterization of high performance fiber reinforced cement composites-HPFRCC." Proceedings of HPFRCC 2, 1-23.

Nanni, A., (1992). "Fatigue behavior of steel fiber reinforced concrete." Cement \& Concrete Composites, 13(4), 239-245.

Nanakorn, P. and Horii, H., (1996). "A fracture-mechanics-based design method for SFRC tunnel linings." Tunnelling and Underground Space Technology, 11(1), 39-43.

Nataraja, M. C., Dhang, N. and Gupta, A. P., (1999). "Stress-strain curves for steel-fiber reinforced concrete under compression." Cement \& Concrete Composites, 21(5-6), 383-390.

Singh, S. and Kaushik, S., (2001). "Flexural fatigue analysis of steel fiber-reinforced concrete." $A C I$ materials Journal, 98(4), 306-312.

Shah, S. P., (1991). "Do fibers increase the tensile strength of cement-based matrixes?" ACI materials Journal, 88(6), 595-602.

Subramaniam, K., Popovics, J. and Shah, S., (2002). "Fatigue fracture of concrete subjected to biaxial stresses in the tensile C-T region." Journal of Engineering Mechanics, ASCE, 128(6), 668-676.

Sumitro, S. and Tsubaki, T., (1998). "Micromechanical fiber pullout model for steel fiber reinforced concrete". J. Materials Conc. Struct., Pavements, JSCE, 40(599), 155-163.

Suryanto, B., Nagai, K. and Maekawa, K., (2010a). "Modeling and analysis of shear-critical ECC members with anisotropic stress and strain fields." Journal of Advanced Concrete Technology, 8(2), 239-258.

Suryanto, B., Nagai, K. and Maekawa, K., (2010b). "Smeared-crack modeling of R/ECC membranes incorporating an explicit shear transfer model." Journal of Advanced Concrete Technology, 8(3), 315-326.

Wecharatan, M. and Shah, S. P., (1983). "A model for predicting fracture resistance of fiber reinforced concrete." Cement and Concrete Research, 13, 819-829.

Zhang, J., Stang, H. and Li, V., (2000). "Experimental study on crack bridging in FRC under uniaxial fatigue tension." Journal of Materials in Civil Engineering, 12(1), 66-73.

Zhang, J. and Stang, H., (1998). "Fatigue performance in flexural of fiber reinforced concrete." $A C I$ Materials Journal, 95(1), 58-67. 